\title{
Interactive comment on "The temperature change shortcut: effects of mid-experiment temperature changes on the deformation of polycrystalline ice" by Lisa Craw et al.
}

\section{Anonymous Referee \#2}

Received and published: 2 January 2021

Review of Craw et al. "The temperature change shortcut: effects of mid-experiment temperature changes on the deformation of polycrystalline ice"

The authors present results from a novel experiment aiming to determine if ice deformation experiments can be sped up by starting deformation tests at higher temperatures, when looking for tertiary strain rates (traditionally very tedious measurements to ascertain). These are exciting results, as they suggest that deformation test times can indeed be decreased by initiating experiments at higher temperatures for relatively high-temperature experiments. Below are some general and specific comments that may improve the paper. 
General Comments: - This is a very well written paper that is clear and easy to follow. Additionally, the figures presented are also easy to interpret, visually pleasing, and appropriately support the text of the manuscript.

- In the Mechanical results section (Section 3.1), the authors state that the tertiary strain rates achieved in the constant-temperature and changing-temperature experiments within each Set are "within the level of variation between the duplicate experiments". From Figure 3 and Table 1, it appears that very similar tertiary strain rates were achieved in all of the $-2 \mathrm{C}$ and $-7 \mathrm{C}$ scenarios, but in the $-10 \mathrm{C}$ scenario it appears that the changing-temperature experiment achieved a lower tertiary strain rate compared to the constant-temperature experiment. However, the authors state in the Discussion that the "Tertiary strain rates at both $-7 \mathrm{C}$ and $-10 \mathrm{C}$ from the changing-temperature experiments agree with those from their equivalent constant-temperature experiments to within the same level of variability..." Please give further justification for this result description and subsequent discussion of the $-10 \mathrm{C}$ scenario.

Specific Comments: - How were the times and amount of added weight chosen to "periodically increase the loads"?

- Why were the samples kept in the rig setup after the experiments ended? Perhaps to not disturb the other experiments going on. Can you give some clarification on why lowering the temperatures to $-18 \mathrm{C}$ and leaving the samples for days does not impact any final microstructure measurements.

- Why do the authors think that the resulting grain size (gs_med) is so different for the two -7C samples (LC004 and LC005), and the two -10C samples (LC021 and LC023)? Similarly, why did the two temperature-change experiment samples in Set 1 (LC006 and LC007) have such a large discrepancy in the resulting grain size? In the Discussion, the authors describe these sets of samples as having indistinguishable microstructure. Therefore, what range of grain size (gs_med) values is considered "indistinguishable", or similar enough? This information will be helpful to the reader

Printer-friendly version

Discussion paper 
while interpreting Figures 4\&5, and Table 1.

Section 1.2, Line 56: unclear what "... this can be delayed significantly beyond the establishment of a quasi-constant strain rate" means. Perhaps consider making this a separate sentence, such as "However, the formation of this steady-state microstructure can occur significantly after the establishment of a quasi-constant strain rate."

Section 1.3, Line 7: remove extra "and" from "... deformed ice and quartz aggregates and quartz veins..."

Section 1.4, Lines 29-33: awkward, long sentence. Consider rewording, especially the transition "...through to tertiary strain, if it is deformed. .."

Section 1.4, Lines 44-45: change the sentence to use either the prepositions "with" or "to" after "compare" (. . to compare X with/to Y...)

Section 2.1, Line 56: change "...frozen into..." to "frozen onto"; also, do you mean aluminum plates instead of "platens"?

Section 2.1, Lines 76-82: These are experimental results and should be moved to the Results section.

Section 3.2, Lines 34-35: It would be nice to see this result visually, instead of taking the authors' word for it.

Appendix A, Line 51: Do the authors mean "G50" here instead of "G60"?

Figure 1 caption: move "(a)" and "(b)" labels to before the panel descriptions.

Figure A1 caption: period at the end of the caption needed.

References: Hammonds \& Baker (2018), Qi et al. (2019) not cited in the text.

Interactive comment on The Cryosphere Discuss., https://doi.org/10.5194/tc-2020-318, 2020. 